\title{
MicroRNA-I 45 downregulation associates with advanced tumor progression and poor prognosis in patients suffering osteosarcoma
}

This article was published in the following Dove Press journal:

OncoTargets and Therapy

6 July 2013

Number of times this article has been viewed

\author{
Mingjie Tangl,\# \\ Lijun Lin',\# \\ Haikang Cai ${ }^{3}$ \\ Jie Tang $^{3}$ \\ Zubin Zhou' \\ 'Department of Orthopedics, \\ Shanghai Sixth People's Hospital, \\ Shanghai Jiaotong University, \\ Shanghai, ${ }^{2}$ Department of \\ Orthopedics, Zhujiang Hospital, \\ The Southern Medical University, \\ Guangzhou, ${ }^{3}$ Department of \\ Orthopedics, Xuhui Central Hospital, \\ Shanghai, People's Republic of China \\ \#These authors contributed equally \\ to this work
}

\begin{abstract}
Purpose: Microribonucleic acid (miRNA)-145 (miR-145) has been identified as a tumor suppressor in several types of human cancers. Especially, miR-145 expression has been found to be significantly decreased in osteosarcoma tissues, and enforced expression of this miRNA could inhibit invasion and angiopoiesis of osteosarcoma cells. However, its clinical significance in osteosarcoma is still unclear. Therefore, the aim of this study was to analyze the association of miR-145 expression with clinicopathologic features and prognosis in patients suffering osteosarcoma.
\end{abstract}

Methods: miR-145 expression was detected by quantitative real-time reverse transcriptase polymerase chain reaction analysis using 166 pairs of osteosarcoma and noncancerous bone tissues. Then, the associations of miR-145 expression with clinicopathological factors or survival of patients suffering osteosarcoma were determined.

Results: The expression levels of miR-145 in osteosarcoma tissues were significantly lower than those in corresponding noncancerous bone tissues $(P<0.0001)$. In addition, miR-145 downregulation more frequently occurred in osteosarcoma specimens with advanced clinical stage $(P=0.003)$ and positive distant metastasis $(P=0.008)$. Moreover, the univariate analysis demonstrated that osteosarcoma patients with low miR-145 expression had poorer overall $(P=0.003)$ and disease-free survival $(P<0.001)$. Furthermore, the multivariate analysis identified low miR-145 expression as an independent prognostic factor for both overall $(P=0.01)$ and disease-free survival $(P=0.008)$.

Conclusion: For the first time, the current data offer convincing evidence that the downregulation of miR-145 may be associated with tumor aggressiveness and tumor metastasis of osteosarcoma, and that this miRNA may be an independent prognostic marker for osteosarcoma patients.

Keywords: osteosarcoma, miR-145, prognosis, overall survival, disease-free survival

\section{Introduction}

Osteosarcoma is the most common primary malignancy, mainly arising from the metaphysis of the long bones of adolescents and young adults. ${ }^{1}$ However, it is a relatively uncommon cancer with an incidence of four to five cases per million (approximately 900 newly diagnosed cases per year in the US). ${ }^{2}$ Despite current treatments combining chemotherapy, surgery, and sometimes radiotherapy, patients with recurrent or metastatic osteosarcomas still have very poor prognosis with $50 \%-60 \%$ of the 5 -year survival rate. ${ }^{3}$ Although recent developments in molecular biology have provided insight into the molecular pathogenesis of osteosarcoma, the fundamental molecular mechanisms underlying the histological heterogeneity, drug resistance, and development of metastasis in this sarcoma have not been fully elucidated. Therefore, the
Correspondence: Zubin Zhou Department of Orthopedics, Shanghai Sixth People's Hospital, Shanghai Jiaotong University, 600 Yishan Road, Shanghai, 200233, People's Republic of China Tel +862164369181

Fax +862164363802

Email zhou_zubin@163.com 
identification of novel molecules as effective drug targets to develop novel alternative therapeutic strategies is very important for improving clinical outcome of patients suffering osteosarcoma.

Microribonucleic acids (miRNAs) are small noncoding RNAs with $18-25$ nucleotides in length. ${ }^{4}$ miRNAs can regulate gene expression through repressing translation and cleaving miRNA by base pairing to the $3^{\prime}$ untranslated region of the target genes. It has been demonstrated that miRNAs play important roles in developmental biology, cellular differentiation programs, and oncogenesis. ${ }^{5}$ In particular, they regulate various cellular processes of tumor cells, including differentiation, progression, apoptosis, and proliferation. miRNAs function as tumor suppressors or oncogenes according to the roles of their target genes. Although recent genome-wide screening using miRNA expression profiles has identified specific miRNA expression patterns which are associated with the biological and clinical properties of cancers, ${ }^{6-8}$ there have been a limited number of studies on the role of miRNAs in osteosarcoma. Here, the focus is on miR-145 because it has been identified as a tumor-suppressive miRNA that is downregulated in several cancer types, including glioma, lung cancer, gastric cancer, colon cancer, prostate cancer, bladder cancer, breast cancer, and ovarian cancer. ${ }^{9-17}$ It can suppress cell growth and invasion by targeting a number of important genes such as TP53, $c-M y c$, MDM2, IRS-1, JAM-A, MUC1, and FSCN1..$^{9-17}$ Interestingly, Fan et al found that miR-145 expression was significantly decreased in osteosarcoma tissues, and enforced expression of this miRNA could inhibit invasion and angiopoiesis of osteosarcoma cell line. ${ }^{18}$ However, the roles of miR-145 in the progression of osteosarcoma and its underlying potential to predict clinical outcome of patients with this sarcoma remain elusive. The aim of this study was to analyze the association of miR-145 expression with clinicopathologic features and prognosis in patients suffering osteosarcoma.

\section{Materials and methods Patients and tissue samples}

This study was approved by the Research Ethics Committee of Shanghai Sixth People's Hospital (Shanghai, People's Republic of China), Zhujiang Hospital (Guangzhou, People's Republic of China), and Xuhui Central Hospital (Shanghai, People's Republic of China). Written informed consent was obtained from all of the patients. All specimens were handled and made anonymous according to the ethical and legal standards.

For quantitative real-time reverse transcriptase polymerase chain reaction (PCR) analysis, 166 primary osteosarcoma and corresponding noncancerous bone tissue samples from the same specimens were collected from the Department of Pathology, Shanghai Sixth People's Hospital, Shanghai Changhai Hospital (Shanghai, People's Republic of China), Shanghai East Hospital (Shanghai, People's Republic of China), Zhujiang Hospital, and Xuhui Central Hospital from 1998 to 2008 . No patients had received blood transfusion, radiotherapy, or chemotherapy before surgery. Clinical stage of these osteosarcoma patients was classified according to the sixth edition of the tumor-node-metastases (TNM) classification of the International Union Against Cancer (UICC). The clinicopathological information of the patients is shown in Table 1.

All 166 osteosarcoma patients received follow-up. The median follow-up was 87 months (range 10-152 months). During the follow-up period, 66 patients (39.8\%) died of disease. Distant metastases developed in 42 patients at a mean of 13.8 months (range 3-46 months) after the original diagnosis. Of these patients, nine had bone metastases and 36 had lung

Table I Correlation of microribonucleic acid-145 expression with clinicopathological features of osteosarcoma

\begin{tabular}{|c|c|c|c|c|}
\hline \multirow[t]{2}{*}{$\begin{array}{l}\text { Clinicopathological } \\
\text { features }\end{array}$} & \multirow[t]{2}{*}{$\begin{array}{l}\text { Number } \\
\text { of cases }\end{array}$} & \multicolumn{2}{|c|}{$\begin{array}{l}\text { Microribonucleic } \\
\text { acid- } 145 \text { expression }\end{array}$} & \multirow[t]{2}{*}{$P$} \\
\hline & & $\begin{array}{l}\text { High } \\
\text { n (\%) }\end{array}$ & $\begin{array}{l}\text { Low } \\
\text { n (\%) }\end{array}$ & \\
\hline \multicolumn{5}{|l|}{ Age } \\
\hline$<55$ years & 72 & $35(48.6)$ & $37(5 \mathrm{I} .4)$ & 0.6 \\
\hline$\geq 55$ years & 94 & $42(44.7)$ & $52(55.3)$ & \\
\hline \multicolumn{5}{|l|}{ Gender } \\
\hline Male & 96 & $46(47.9)$ & $50(52.1)$ & 0.6 \\
\hline Female & 70 & $31(44.3)$ & $39(55.7)$ & \\
\hline \multicolumn{5}{|l|}{ Tumor size } \\
\hline$>8 \mathrm{~cm}$ & 88 & $4 \mid(46.6)$ & $47(53.4)$ & 1.0 \\
\hline$\leq 8 \mathrm{~cm}$ & 78 & $36(46.2)$ & $42(53.8)$ & \\
\hline \multicolumn{5}{|l|}{ Anatomic location } \\
\hline Tibia/femur & 103 & $50(48.5)$ & $53(51.5)$ & 0.5 \\
\hline Elsewhere & 63 & $27(42.9)$ & $36(57.1)$ & \\
\hline \multicolumn{5}{|c|}{ Serum level of lactate dehydrogenase } \\
\hline Elevated & 90 & $45(50.0)$ & $45(50.0)$ & 0.5 \\
\hline Normal & 76 & $32(42.1)$ & $44(57.9)$ & \\
\hline \multicolumn{5}{|c|}{ Serum level of alkaline phosphatase } \\
\hline Elevated & 108 & $50(46.3)$ & $58(53.7)$ & 1.0 \\
\hline Normal & 58 & $27(46.6)$ & $31(53.4)$ & \\
\hline \multicolumn{5}{|l|}{ Clinical stage } \\
\hline IIA & 68 & $49(72.1)$ & $19(27.9)$ & $0.003 *$ \\
\hline IIB/III & 98 & $28(28.6)$ & $70(7 \mid .4)$ & \\
\hline \multicolumn{5}{|l|}{ Distant metastasis } \\
\hline Absent & 124 & $67(54.0)$ & $57(46.0)$ & $0.008 *$ \\
\hline Present & 42 & $10(23.8)$ & $32(76.2)$ & \\
\hline \multicolumn{5}{|c|}{ Response to chemotherapy } \\
\hline Good & 68 & $33(48.5)$ & $35(5 \mathrm{I} .5)$ & 0.6 \\
\hline Poor & 98 & $44(44.9)$ & $54(55.1)$ & \\
\hline
\end{tabular}

Note: *statistically significant $P$-values. 
metastases (three patients had both bone and lung metastases). The median overall and disease-free survival of patients was 31 months (95\% confidence interval [CI], 30.1-42.9 months) and 25 months (95\% CI 23.7-35.2 months), respectively.

\section{Quantitative reverse transcriptase PCR assay}

The expression levels of miR-145 in osteosarcoma and corresponding noncancerous tissues were detected by quantitative reverse transcriptase PCR assay. Briefly, total RNA was extracted from tissues using TRIzol ${ }^{\circledR}$ (Life Technologies, Carlsbad, CA, USA) according to the manufacturer's protocol. Then, miRNA expression levels were quantitated using a MicroRNA Assay Kit (Life Technologies) according to the manufacturer's protocol. The two-step protocol involves reverse transcription with an miRNA-specific primer to convert miRNA to complementary DNA, followed by real-time quantitative PCR with TaqMan ${ }^{\circledR}$ probes. The universal small nuclear RNA U6 (RNU6B) was used as an endogenous control for miRNAs. Each sample was examined in triplicate and the amounts of the PCR products produced were nonneoplasticized to RNU6B.

\section{Statistical analysis}

The software of SPSS 13.0 for Windows (SPSS Inc, Chicago, IL, USA) and SAS 9.1 (SAS Institute, Cary, NC, USA) was used for statistical analysis. Continuous variables were expressed as mean \pm standard deviation (SD). The Chi-squared test was used to show differences of categorical variables. Patient survival and their differences were determined by Kaplan-Meier method and logrank test. Cox regression (proportional hazard model) was adopted for multivariate analysis of prognostic factors. Differences were considered statistically significant when $P<0.05$.

\section{Results}

\section{Downregulation of miR-I45}

\section{in human osteosarcoma tissues}

The expression levels of miR-145 were detected in 166 pairs of osteosarcoma and corresponding noncancerous bone tissues normalized to RNU6B. As shown in Figure 1, the expression levels of miR-145 in osteosarcoma tissues were found to be distinctly decreased compared to noncancerous bone tissues. The results showed that the relative level of miR-145 expression in osteosarcoma tissues (mean \pm SD: $2.4 \pm 1.3$ ) was significantly lower than that in corresponding noncancerous bone tissues (mean $\pm \mathrm{SD}$ : $4.3 \pm 1.2$; $P<0.0001)$.

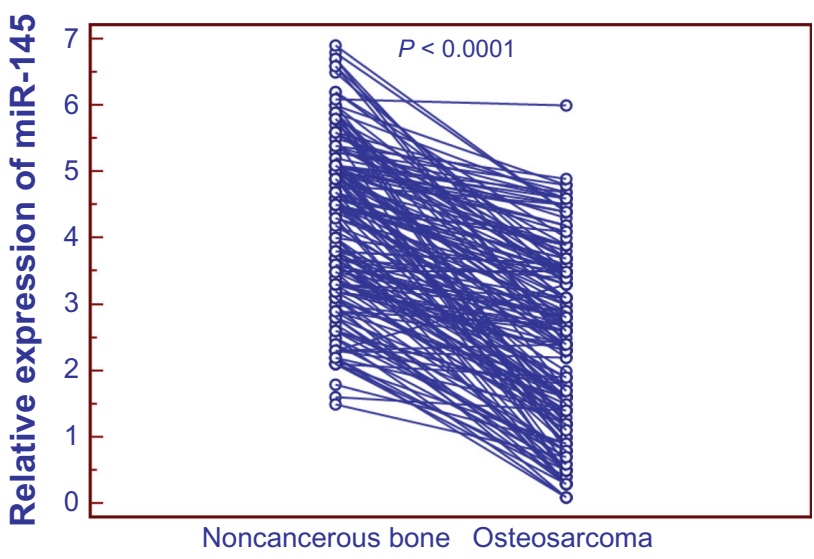

Figure I miR-145 expressions in I66 pairs of osteosarcoma and corresponding noncancerous bone tissues were respectively detected by real-time quantitative reverse transcriptase polymerase chain reaction assay.

Note: The expression levels of miR-145 in osteosarcoma tissues were significantly lower than those in corresponding noncancerous bone tissues $(P<0.000 \mathrm{I})$. Abbreviation: miR-145, microribonucleic acid-145.

\section{Downregulation of miR-I45 associates with advanced clinicopathological features of osteosarcoma}

The associations of miR-145 expression with various clinicopathological parameters of osteosarcoma tissues were analyzed. The median of miR-145 expression levels in all 166 patients with osteosarcoma was 2.6. The patients were divided into two groups according to their expression levels of miR-145, using its median as a cutoff: high miR-145 expression group $(n=77$; mean \pm SD: $3.6 \pm 0.7)$ and low miR-145 expression group $(n=89$; mean \pm SD: $1.4 \pm 0.7)$. As shown in Table 1, miR-145 was significantly downregulated in osteosarcoma patients with advanced clinical stage $(P=0.003)$ and positive distant metastasis $(P=0.008)$ as compared to those with low clinical stage and without distant metastasis. No significant difference was observed between the expression of miR-145 and patients' age, gender, tumor size, anatomic location, serum levels of lactate dehydrogenase and alkaline phosphatase, and response to chemotherapy.

\section{Downregulation of miR-I45 confers poor prognosis in patients with osteosarcomas}

Using the Kaplan-Meier method and logrank test, the overall $(P=0.003$; Figure 2A) and disease-free survival $(P<0.001$; Figure 2B) of osteosarcoma patients with low miR-145 expression were both significantly shorter than those with high miR-145 expression. Besides, the survival benefits were also found in those with smaller tumor size (both $P=0.03$ ), higher clinical stage ( $P=0.01$ and $P=0.006$, respectively), 

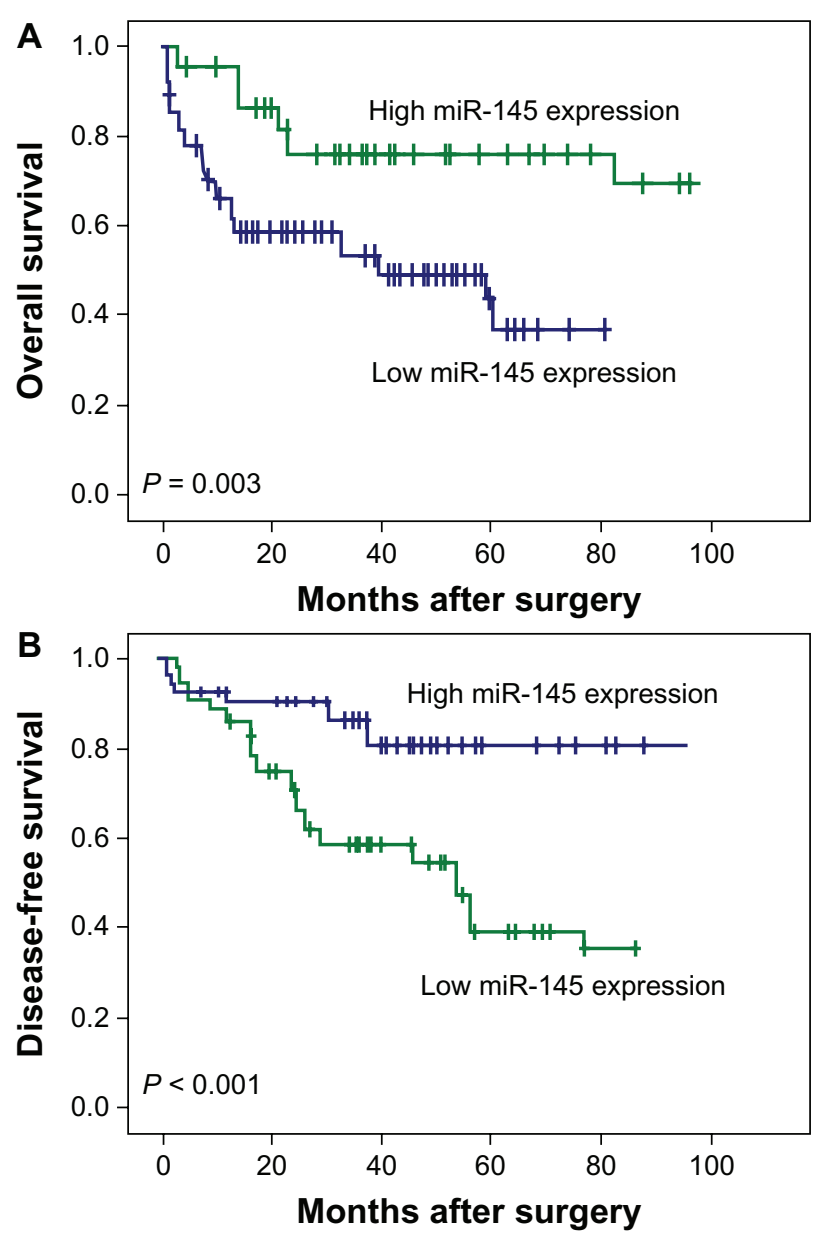

Figure 2 (A) Overall and (B) disease-free survival curves for two groups defined by low and high expression of miR-145 in patients with osteosarcoma.

Notes: The patients with low miR-145 expression had a significantly worse 5-year overall $(P=0.003)$ and disease-free $(P<0.001)$ survival rate than those with high miR-145 expression.

Abbreviation: miR-145, microribonucleic acid-145.

without distant metastasis $(P=0.008$ and $P=0.003$, respectively), and better response to chemotherapy (both $P=0.02$ ) for overall and disease-free survival.

Multivariate Cox regression analysis enrolling abovementioned significant parameters revealed that miR-145 expression (relative risk [RR] 3.6; 95\% CI 1.1-8.9; $P=0.01$ ), clinical stage (RR 2.3; 95\% CI 1.1-7.0; $P=0.02$ ), distant metastasis status (RR 3.7; 95\% CI 1.9-9.8; $P=0.01$ ), and response to chemotherapy (RR 2.6; 95\% CI 1.5-8.2; $P=0.02$ ) were independent prognostic markers for overall survival of patients with osteosarcoma (Table 2). Turning to disease-free survival, miR-145 expression (RR 4.1; 95\% CI 1.2-10.8; $P=0.008)$, clinical stage (RR $1.8 ; 95 \%$ CI $0.7-8.1 ; P=0.03$ ) and metastasis status (RR 2.9; 95\% CI 1.3-10.6; $P=0.02$ ) were also independent prognostic markers for disease-free survival of patients with osteosarcoma (Table 2).
Table 2 Multivariate survival analysis of overall survival and disease-free survival in 166 patients with osteosarcoma

\begin{tabular}{|c|c|c|c|c|c|c|}
\hline \multirow[t]{2}{*}{ Variables } & \multicolumn{3}{|c|}{ Overall survival } & \multicolumn{3}{|c|}{ Disease-free survival } \\
\hline & $\mathbf{R R}$ & $95 \% \mathrm{Cl}$ & $\boldsymbol{P}$ & $\mathbf{R R}$ & $95 \% \mathrm{Cl}$ & $\mathbf{P}$ \\
\hline $\begin{array}{l}\text { Microribonucleic } \\
\text { acid-145 expression }\end{array}$ & 3.6 & $1.1-8.9$ & 0.01 & 4.1 & $1.2-10.8$ & 0.008 \\
\hline Clinical stage & 2.3 & I.I-7.0 & 0.02 & 1.8 & $0.7-8.1$ & 0.03 \\
\hline $\begin{array}{l}\text { Distant metastasis } \\
\text { status }\end{array}$ & 3.7 & $1.9-9.8$ & 0.01 & 2.9 & $1.3-10.6$ & 0.02 \\
\hline $\begin{array}{l}\text { Response to } \\
\text { chemotherapy }\end{array}$ & 2.6 & I.5-8.2 & 0.02 & 0.9 & $0.3-1.7$ & 0.6 \\
\hline
\end{tabular}

Abbreviations: $\mathrm{Cl}$, confidence interval; $\mathrm{RR}$, relative risk.

\section{Discussion}

miRNAs have been demonstrated to play an important role in cancer cell growth and migration; however, their potential roles in osteosarcoma remain largely uncharacterized. In the current study, it was found that miR-145 was downregulated in human osteosarcoma tissues compared with noncancerous bone tissues. It was also found that the decreased expression of miR-145 in osteosarcoma tissues was significantly correlated with aggressive clinicopathological features. Moreover, the results of Kaplan-Meier analyses shown that osteosarcoma tissues with low miR-145 expression tend to have shorter overall and disease-free survival. Finally, the multivariate analysis clearly demonstrated that the low expression of miR-145 was a statistically significant risk factor affecting both overall and disease-free survival in osteosarcoma patients, suggesting that miR-145 expression could be a valuable marker of osteosarcoma progression and prognosis. To the authors' knowledge, this is the first study to investigate the clinical significance of miR-145 in a large number of osteosarcoma patients.

In 2002, miR-145 was originally identified in mouse heart muscle. ${ }^{19}$ In 2003, Michael et al subsequently verified its expression in humans and demonstrated the significantly reduced levels of miR-145 in precancerous and neoplastic colorectal tissue. ${ }^{20}$ The sequence of human miR-145 was predicted based on homology to a verified miRNA from mouse. It has been demonstrated to regulate cell apoptosis, proliferation, neural development, and stem cell differentiation. Under the physiological condition, miR-145 is overexpressed in germline and mesoderm-derived tissues such as uterus, ovary, prostate, testis, spleen, and heart, ${ }^{21}$ while its expression is distinctly reduced in many human cancers such as colon cancer, breast cancer, lung cancer, hepatocellular carcinoma, and ovary and prostate carcinomas. ${ }^{9-17}$ miR-145 functions as a tumor suppressor by targeting a number of 
oncogenic genes. For example, miR-145 suppresses tumor growth and angiogenesis by targeting insulin receptor substrate-1, p70S6K1, and c-Myc; ${ }^{9-11,13}$ miR-145 impacts migration, invasion, and metastasis by targeting Fli-1 and mucin-1, respectively; ${ }^{12,14,15}$ and miR-145 affects p53-mediated cell cycle arrest by targeting p21. ${ }^{16,17}$ In addition to its targeting genes, recent studies also have investigated the relationship between miR-145 and several cancer-related signal pathways. For example, miR-145 exhibits its antitumor effect in human colon cells by regulating p21-activated kinase- 4 via the mitogen-activated protein kinase pathway; ${ }^{22}$ Ras activation may lead to miR-145 repression and miR-145 may activate targets $K R A S$ and $R R E B 1 ;{ }^{23}$ and miR-145 can be negatively regulated by CCAAT-enhancer-binding-protein- $\beta$ through the Akt pathway. ${ }^{24}$ These findings highlight the significance of miR-145 as a target for cancer diagnosis and therapy.

The current data showed the downregulation of miR-145 in osteosarcoma tissues, which is consistent with the previous study of Fan et al. ${ }^{18}$ This consistency prompted the authors to investigate the clinical significance it has in osteosarcoma. It is evident from the results that the reduced expression of miR-145 more frequently occurs in osteosarcoma tissues with advanced clinical stage and the presence of distant metastasis. This led to the hypothesis that miR-145 might be related to the clinical outcome of patients with osteosarcoma. Both the univariate and multivariate survival analyses demonstrated that loss of miR-145 may be associated with shorter overall and disease-free survival in osteosarcoma, which was also consistent with the prognostic value of miR-145 in other human malignancies, such as prostate cancer, ${ }^{25}$ colon cancer, ${ }^{26}$ and esophageal cancer. ${ }^{27}$

\section{Conclusion}

For the first time, the current data offer convincing evidence that the downregulation of miR-145 may be associated with tumor aggressiveness and tumor metastasis of osteosarcoma, and that this miRNA may be an independent prognostic marker for osteosarcoma patients. Fan et al have indicated that miR-145 could inhibit tumor invasion and metastasis of osteosarcoma cell lines by binding to the $3^{\prime}$ untranslated region of vascular endothelial growth factor messenger RNA specifically, subsequently reducing the expression of vascular endothelial growth factor. ${ }^{18}$ However, the molecular mechanisms underlying miR-145 and the regulation of osteosarcoma carcinogenesis have not been fully elucidated. Therefore, this study is hypothesis generating, and further prospective analysis is worth doing.

\section{Disclosure}

The authors report no conflicts of interest in this work.

\section{References}

1. Ottaviani G, Jaffe N. The epidemiology of osteosarcoma. Cancer Treat Res. 2009;152:3-13.

2. Teicher BA. Searching for molecular targets in sarcoma. Biochem Pharmacol. 2012;84(1):1-10.

3. Gorlick R. Current concepts on the molecular biology of osteosarcoma. Cancer Treat Res. 2009;152:467-478.

4. Osman A. MicroRNAs in health and disease - basic science and clinical applications. Clin Lab. 2012;58(5-6):393-402.

5. Izzotti A. Molecular medicine and the development of cancer chemopreventive agents. Ann NY Acad Sci. 2012;1259:26-32.

6. Kong YW, Ferland-McCollough D, Jackson TJ, Bushell M. MicroRNAs in cancer management. Lancet Oncol. 2012;13(6):e249-e258.

7. Cho WC. Great potential of miRNAs as predictive and prognostic markers for cancer. Expert Rev Mol Diagn. 2012;12(4):315-318.

8. Kobayashi E, Hornicek FJ, Duan Z. MicroRNA involvement in osteosarcoma. Sarcoma. 2012;2012:359739.

9. Xu Q, Liu LZ, Qian X, et al. MiR-145 directly targets p70S6K1 in cancer cells to inhibit tumor growth and angiogenesis. Nucleic Acids Res. 2012; 40(2):761-774.

10. Speranza MC, Frattini V, Pisati F, et al. NEDD9, a novel target of miR-145, increases the invasiveness of glioblastoma. Oncotarget. 2012;3(7): 723-734.

11. Yun SJ, Jeong P, Kim WT, et al. Cell-free microRNAs in urine as diagnostic and prognostic biomarkers of bladder cancer. Int J Oncol. 2012; 41(5):1871-1878.

12. Huang S, Guo W, Tang Y, Ren D, Zou X, Peng X. miR-143 and miR-145 inhibit stem cell characteristics of PC-3 prostate cancer cells. Oncol Rep. 2012;28(5):1831-1837.

13. Bauer KM, Hummon AB. Effects of the miR-143/-145 microRNA cluster on the colon cancer proteome and transcriptome. J Proteome Res. 2012;11(9):4744-4754.

14. Miles GD, Seiler M, Rodriguez L, Rajagopal G, Bhanot G. Identifying microRNA/mRNA dysregulations in ovarian cancer. BMC Res Notes. 2012;5:164.

15. Gao P, Xing AY, Zhou GY, et al. The molecular mechanism of microRNA-145 to suppress invasion-metastasis cascade in gastric cancer. Oncogene. 2013;32(4):491-501.

16. Lu Y, Govindan R, Wang L, et al. MicroRNA profiling and prediction of recurrence/relapse-free survival in stage I lung cancer. Carcinogenesis. 2012;33(5):1046-1054.

17. Zhang J, Sun Q, Zhang Z, Ge S, Han ZG, Chen WT. Loss of microRNA$143 / 145$ disturbs cellular growth and apoptosis of human epithelial cancers by impairing the MDM2-p53 feedback loop. Oncogene. 2013; 32(1):61-69.

18. Fan L, Wu Q, Xing X, Wei Y, Shao Z. MicroRNA-145 targets vascular endothelial growth factor and inhibits invasion and metastasis of osteosarcoma cells. Acta Biochim Biophys Sin (Shanghai). 2012;44(5): 407-414.

19. Lagos-Quintana M, Rauhut R, Yalcin A, Meyer J, Lendeckel W, Tuschl T. Identification of tissue-specific microRNAs from mouse. Curr Biol. 2002;12(9):735-739.

20. Michael MZ, O'Connor SM, van Holst Pellekaan NG, Young GP, James RJ. Reduced accumulation of specific microRNAs in colorectal neoplasia. Mol Cancer Res. 2003;1(12):882-891.

21. Kim SJ, Oh JS, Shin JY, et al. Development of microRNA-145 for therapeutic application in breast cancer. J Control Release. 2011;155(3): 427-434.

22. Wang Z, Zhang X, Yang Z, et al. MiR-145 regulates PAK4 via the MAPK pathway and exhibits an antitumor effect in human colon cells. Biochem Biophys Res Commun. 2012;427(3):444-449. 
23. Kent OA, Fox-Talbot K, Halushka MK. RREB1 repressed miR143/145 modulates KRAS signaling through downregulation of multiple targets. Oncogene. 2013;32(20):2576-2585.

24. Sachdeva M, Liu Q, Cao J, Lu Z, Mo YY. Negative regulation of miR145 by C/EBP- $\beta$ through the Akt pathway in cancer cells. Nucleic Acids Res. 2012;40(14):6683-6692.

25. Schaefer A, Jung M, Mollenkopf HJ, et al. Diagnostic and prognostic implications of microRNA profiling in prostate carcinoma. Int J Cancer. 2010;126(5):1166-1176.
26. Wang CJ, Zhou ZG, Wang L, et al. Clinicopathological significance of microRNA-31, -143 and -145 expression in colorectal cancer. Dis Markers. 2009;26(1):27-34.

27. Liu R, Liao J, Yang M, et al. The cluster of miR-143 and miR-145 affects the risk for esophageal squamous cell carcinoma through co-regulating fascin homolog 1. PLoS One. 2012;7(3):e33987.

\section{Publish your work in this journal}

OncoTargets and Therapy is an international, peer-reviewed, open access journal focusing on the pathological basis of all cancers, potential targets for therapy and treatment protocols employed to improve the management of cancer patients. The journal also focuses on the impact of management programs and new therapeutic agents and protocols on

\section{Dovepress}

patient perspectives such as quality of life, adherence and satisfaction. The manuscript management system is completely online and includes a very quick and fair peer-review system, which is all easy to use. Visit http://www.dovepress.com/testimonials.php to read real quotes from published authors.

Submit your manuscript here: http://www.dovepress.com/oncotargets-and-therapy-journal 